\title{
Importance of Flexibility in Manufacturing Systems
}

\author{
Héctor Kaschel C., Luis Manuel Sánchez y Bernal
}

\begin{abstract}
Flexibility refers to the ability of a manufacturing system to respond cost effectively and rapidly to changing production needs and requirements. This capability is becoming increasingly important for the design and operation of manufacturing systems, as these systems do operate in highly variable and unpredictable environments.

It is important to system designers and managers to know of different levels of flexibility and / or determine the amount of flexibility required to achieve a certain level of performance. This paper shows several representations schemes for product flexibility and discusses the usefulness and limitations of each.

Keywords: Flexibility, Fuzzy Logic, Disjunctive Graph, Constraints Temporal Network, Complexity in Manufacturing, Non Lineal Mathematic Programming
\end{abstract}

\section{Introduction}

Present market demands require that Manufacturing Systems develop their activities under a dynamic and uncertain production environment (Calinescu et al., 2003). To understand the problems affecting Flexible Manufacturing Systems (FMS), it is necessary to incorporate the concept of flexibility in the scheduling (Deshmukh et al. 2002). Flexibility constitutes a strategic topic in decision making to give quick and efficient answers to the demands of the national and international markets (Pelaez, and Ruiz, 2004).

In order to define the flexibility in FMS, the scientific literature reports a wide variety of concepts. For example, flexibility for Sethi and Sethi (Sethi and Sethi, 1992) represents the capacity of FMS to modify manufacturing resources to produce different products efficiently maintaining an acceptable quality.

For Gupta (Gupta, 2004), it is necessary to make the difference between flexibility inherent to the FMS and flexibility required during the production cycle. The first one is defined as the capacity of resources and human operators to implement decisions of the factory company. While required flexibility is defined by the changes in the environment that surrounds the FMS and for the implementation of new manufacturing strategies. Starting from these differences, it is possible to determine the flexibility type and measure of the FMS quantitatively.

For (Benjaafar and Ramakrishman, 1996), it is important to differentiate the types of flexibility in the FMS. They define the flexibility of the FMS as product flexibility and process flexibility. The first one refers to the variety of factory options for a certain product. While process flexibility is defined as a characteristic of an industrial process to operate under diverse dynamic conditions of operation. Both general flexibilities can be grouped hierarchically as shown in the figure 1.

The classification proposed by (Benjaafar and Ramakrishman, 1996) shows flexibility as a consequence of the physical and logical characteristics that the manufacturing system presents To quantify the operative flexibility of job $\mathrm{i}$, a function is defined starting from the time of dependent configuration of the sequence between operations:

$$
\Phi\left(p_{i}\right)=\frac{-\sum_{j=1}^{N_{i}} \sum_{l=1}^{k_{i j}}\left(\frac{\frac{1}{s_{i j l}}}{\sum_{l=1}^{k_{i j}} \frac{1}{s_{i j l}}}\right) \log \left(\frac{\frac{1}{s_{i j l}}}{\sum_{l=1}^{k_{i j}} \frac{1}{s_{i j l}}}\right)}{N_{i}}
$$

where: 


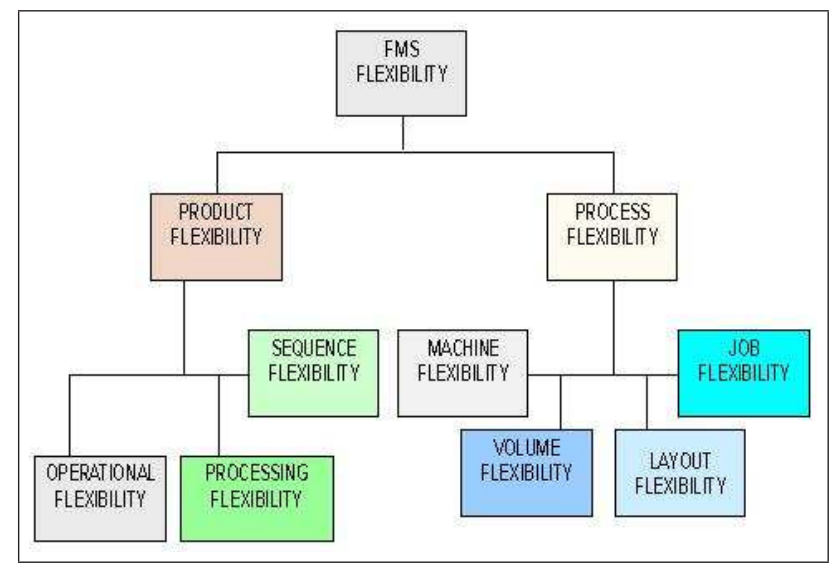

Figure 1: Classification of the FMS Flexibility

- $s_{i j l}$ represents the time of configuration of job $i$ during the commutation of the operation $j$ to the operation $l$.

- $N_{i}$ represents the total number of operations associated to job $i$.

In the present paper different models were analyzed as described in several studies dealing with product flexibility in FMS.

\section{Product flexibility}

In all FMS, each job or jobs group is associated to a certain scheduling, consisting in an orderly sequence of operations to be executed in a group of machines. An example of scheduling of the FMS is illustrated in figure 2(a).

If we incorporate conditions of flexibility to the scheduling (operational flexibility), an operation can be carried out in other machines. These, in general, can be different or they can have different operational levels (figure 2 (b)).

The classification of the operations can also be varied (sequence flexibility), where some operations should be carried out in sequence while others don't have this restriction. An example of this type of flexibility is shown in figure 2(c), where operations 2 and 3 can be carried out in any order after executing operation 1.

Finally, in a highly flexible FMS, a job can have a group of schedulings (processing flexibility). This means that the group of operations required to manufacture a job can be variable and depending on the operation conditions of the FMS. For example, in figure 2(d), operations 1 and 2 can be replaced by the operation 5 while operation 3 can be divided in operations 6 and 7 .

\section{Analysis models for product flexibility}

The product flexibility constitutes an important aspect in the functionality of the FMS, where the variability in the scheduling and the operability of the machines allow several alternatives to process the jobs. In what follows some models of analysis of the product flexibility are presented. 


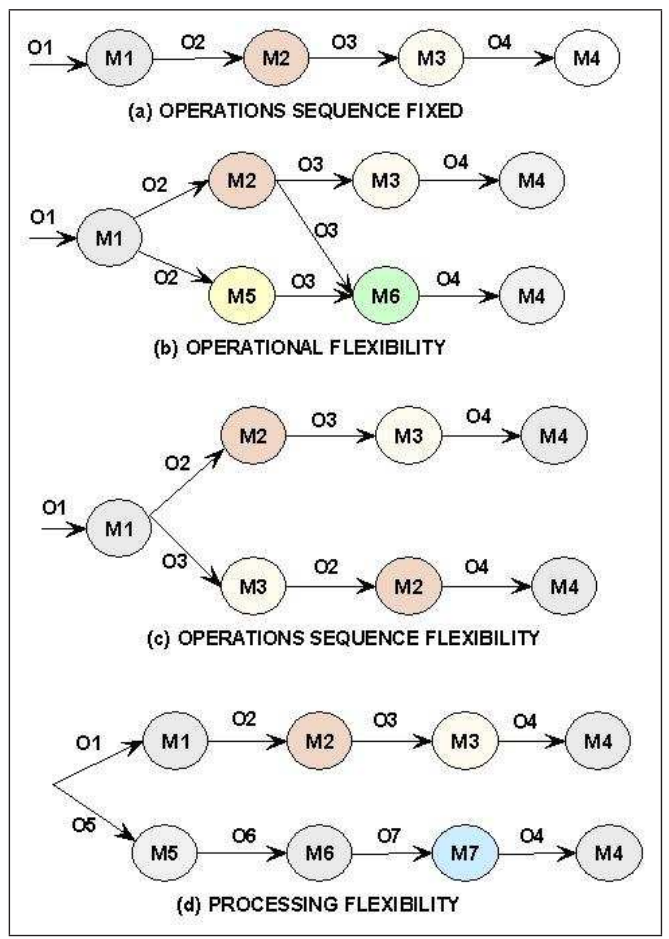

Figure 2: Types of Product Flexibility

\subsection{Disjunctive Graph}

It is a model of directed graph, whose nodes have a certain weight assigned. Mathematically it is expressed as 3 - tupla (Benjaafar and Ramakrishman, 1996):

$$
G=(N, A, E)
$$

where:

- $\mathrm{N}$ : represents the group of nodes that represent the operations. In this model are defined two particular nodes, called initial and final. The positive weight assigned to each node represents the processing time of the corresponding operation. The initial node is connected with the first operation of each job, and in the same manner the last operation of each job is connected with the final node.

- A: represents the group of conjunctive directed arcs that indicate the precedence restrictions among the operations of each job.

- E: represents the group of disjunctive directed arcs that indicate the restrictions of machines operative capacity.

To illustrate the application of the disjunctive graph, an example of scheduling of 4 different jobs is presented $\left(J_{1}, J_{2}, J_{3}, J_{4}\right)$ in three types of machines $\left(M_{1}, M_{2}, M_{3}\right)$. The distribution of processing times and assignment of machines is shown in table 1, starting from this information we proceed to generate the disjunctive graph of figure 3, that shows the precedence restrictions between the operations (conjunctive arcs) and the flexibility in the machines operative sequence (disjunctive arcs). 


\begin{tabular}{|l|ccc|ccc|}
\hline & \multicolumn{3}{|c|}{ Processing time } & \multicolumn{3}{c|}{ Machines assignment } \\
\cline { 2 - 7 }$J_{1}$ & $M_{1}$ & $M_{2}$ & $M_{3}$ & 1 & 2 & 3 \\
$J_{2}$ & 3 & 9 & 2 & $M_{1}$ & $M_{2}$ & $M_{3}$ \\
$J_{3}$ & 1 & 10 & 7 & $M_{3}$ & $M_{1}$ & $M_{2}$ \\
$J_{4}$ & 7 & 4 & $M_{3}$ & $M_{2}$ \\
\hline
\end{tabular}

Table 1: Machines assignment and processing times

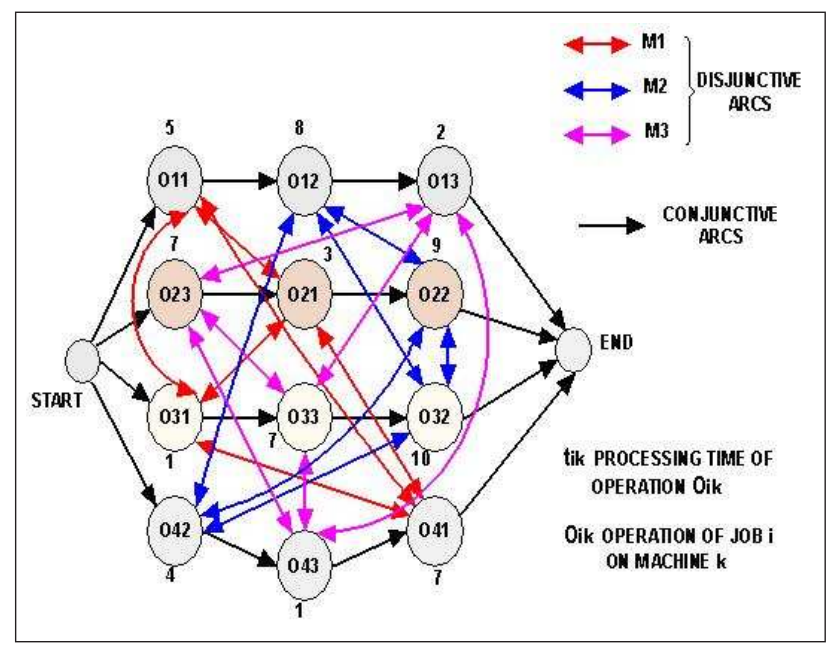

Figure 3: Disjunctive Graph generated by table 1

\subsection{Temporal Constraints Network}

It is a model of directed graph that allows the representation of temporary constraints assigned to the precedence relationships between operations that are part of a scheduling of a FMS. The nodes of the graph represent the operations and the arcs have associated temporary intervals to denote the temporary constraints that exist between two nodes. All the times are calculated starting from of the scheduling initial state. The development of the network is based mathematically from Disjunctive Metric Temporal Algebra (Alfonso, 2001).

This methodology is used to solve scheduling problems characterized from a group of temporary constraints, consequently, the techniques developed in areas like Temporary Reasoning and Constraints Satisfaction can be applied. In general, two techniques are used: Closure and Constraint Satisfaction Problem (CSP) (Barber, 2000).

Closure is a deductive process by means of which new constraints are inferred, starting from existing constraints. It also allows to detect possible inconsistencies (value of the variables that don't lead to any solution) that can be eliminated. The main advantage of the closure techniques consists in the reduction of the search space. Therefore, the closure is used fundamentally as a previous step to a process of search of solutions.

Techniques of Constraints Satisfaction Problem constitutes a process of search of solutions by means of the successive assignment of values to the variables that are part of the constraints of the problem. In a process of Constraints Satisfaction Problem, it is important to use different heuristics to make the search process more efficient (Beck and Fox, 2000).

To illustrate the use of the Temporary Constraints Network, a distribution of temporary constraints is presented among four operations of a FMS (see table 2).

Starting from the information in table 2, we proceed to establish the group of operations $(O)$, the 


\begin{tabular}{|c|c|c|c|c|}
\hline & $O_{1}$ & $O_{2}$ & $O_{3}$ & $O_{4}$ \\
\hline$O_{0}$ initial state & $(10,20)$ & $(10,20)$ & $(20,50)$ & $(60,70)$ \\
\hline$O_{1}$ & & $(30,40),(60,60)$ & $(10,30),(40,40)$ & $(40,60)$ \\
\hline$O_{2}$ & & & & $(0,30)$ \\
\hline$O_{3}$ & & $(10,20)$ & & $(20,30),(40,50)$ \\
\hline
\end{tabular}

Table 2: Precedence Temporary Constraints between operations

group of precedence relationships between operations $(P)$ and the group of temporal relationships between operations $(R)$. Finally, starting from the definitions of the groups we proceed to generate the corresponding Graph of Temporal Constraints (see figure 4):

$$
\begin{gathered}
G=(O, P) \\
O=\left(O_{0}, O_{1}, O_{2}, O_{3}, O_{4}\right) \\
P=\left\{\begin{array}{c}
\left(0_{0}, O_{1}\right),\left(0_{0}, O_{2}\right),\left(0_{0}, O_{3}\right),\left(0_{0}, O_{4}\right) \\
\left(0_{1}, O_{2}\right),\left(0_{1}, O_{3}\right),\left(0_{1}, O_{4}\right),\left(0_{2}, O_{4}\right) \\
\left(0_{3}, O_{2}\right),\left(0_{3}, O_{4}\right)
\end{array}\right\} \\
R=\left\{\begin{array}{c}
\left(O_{0}(10,20) O_{1}\right),\left(O_{0}(10,20) O_{2}\right), \\
\left.O_{0}(20,50) O_{3}\right),\left(O_{0}(60,70) O_{4},\right. \\
\left(O_{1}(30,40)(60,60) O_{2}\right), \\
\left(O_{1}(10,30)(40,40) O_{3}\right),\left(O_{1}(40,60) O_{4}\right), \\
\left(O_{2}(0,30) O_{4}\right),\left(O_{3}(10,20) O_{2}\right) \\
\left(O_{3}(20,30)(40,50) O_{4}\right)
\end{array}\right\}
\end{gathered}
$$

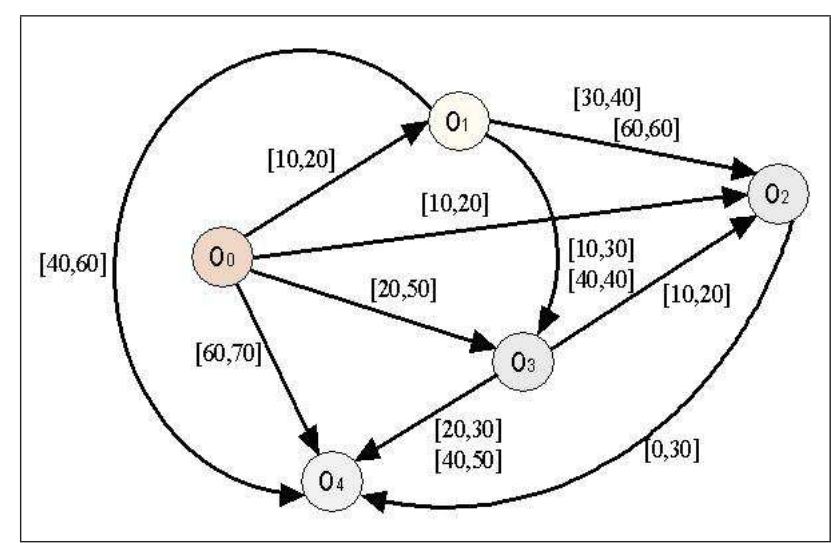

Figure 4: Temporary Constraints Network generated by Table 2

\subsection{Model based on Mathematical Programming}

Chandra and Tombak (Chandra and Tombak, 1993) propose a operations sequence flexibility model, based on the theory of lineal programming. The model incorporates the concepts of machines reliability and machines processing capacity. Machine reliability is defined as the probability of an operation to be executed by a machine in a certain interval of time. Machine processing capacity, represents the total number of jobs that a machine can execute in a interval of time. 
The FMS base for the development of the model consists of a finite group of machines that can present independent fault events. The model is formulated considering the jobs flow on a fixed scheduling and it is expressed mathematically as:

$$
R F=\max \sum_{i} \sum_{h} c_{i h} x_{i h}
$$

subject to the following constraints:

$$
\begin{gathered}
\sum_{i} \sum_{h \mid b_{i h k}=1} \frac{t_{i k}}{P_{k}} x_{i h} \leq T_{k}, \forall k \\
\sum_{h} x_{i h}>d_{i}, \forall i \\
x_{i h} \geq 0, \forall i, h
\end{gathered}
$$

where,

- $t_{i k}$ represents the processing time of job $i$ in machine $k$;

- $T_{k}$ represents the total processing time of machine $k$;

- $P_{k}$ represents the probability that machine $k$ is operative in a certain time;

- $b_{i h k}$ represents the possibility that job $i$ is processed in machine $k$ along route $h$;

- $C_{h}$ represents the factor of cost of job $i$ when processed in route $h$. This factor depends on the reliability machines located along route $h$;

- $d_{i}$ represents the minimum demand of job $i$ and,

- $x_{i h}$ represents the flow of job $i$ on route $h$.

The model represents the processing capacity of each machine considering its reliability, where the factor $\frac{t_{i k}}{P_{k}}$ represents the time required to process job $i$ in machine $k$. The model considered $n+m$ constraints and $m\left(1+\sum_{k=1}^{n-2} k ! C_{k}^{n-2}\right)$ variables in case of full machines connections.

\subsection{Model based on Fuzzy Logic}

In (Tsourveloudis and Phillis, 1998) and (Fortemps, 2000), analyze the flexibility starting from a methodology based on the knowledge that the human expert has on the structural and dynamic behavior of the FMS. For (Tsourveloudis and Phillis, 1998) the flexibility is represented by means of a group of diffuse linguistic rules of the type:

$$
\text { IF }\langle\text { antecedent fuzzy }\rangle \text { THEN }\langle\text { consequent fuzzy }\rangle
$$

This proposal is based on the fuzzy behavior that the FMS presents, in cases of concurrence and synchronization of operations or fault machines events. For (Tsourveloudis and Phillis, 1998), a FMS presents seven different types of flexibility:

1. Materials Manipulation System Flexibility: It measures the capacity of the system to transfer several jobs types efficiently between two neighboring points.

2. Product Flexibility: It measures the capacity of the system to produce mixed jobs. 
3. Operative flexibility: It measures the capacity to modify the sequence of operations to produce a job.

4. Process Flexibility: It measures the capacity of the system to produce different jobs without modifying the FMS structure.

5. Volume Flexibility: It measures the operative capacity of the FMS for different levels of productivity.

6. Scalability Flexibility: It measures the cost or time required to enlarge a FMS.

7. Labor Flexibility: It measures the operator's capacity to develop different operative tasks of FMS.

Each one of these types of flexibility is expressed by means of a fuzzy rule that represents the expert's knowledge about this flexibility. The fuzzy rule is expressed mathematically as:

$$
\text { IF } F_{1} \text { is } A_{1} A N D \ldots \text { AND } F_{N} \text { is } A_{N} \text { THEN } F_{M F} \text { is MF }
$$

where: $A_{i}=\{$ Low,About_Low,Average,About_High,High $\}$ represents the group of linguistic values for the flexibility parameter. For example, the following fuzzy rule expresses the Operative Flexibility of the FMS:

$$
\text { IF } C_{0} \text { is } T_{C_{0}} \text { AND } S_{B} \text { is } T_{S_{B}} \text { THEN } F_{R} \text { is } T_{F_{R}}
$$

For this rule, $C_{0}$ represents the number of operations common to machines group and $S_{B}$ represents the FMS capacity to route a jobs group under conditions of machines faults.

\section{Conclusions}

The models described in this paper define a set of basic principles that should satisfy all parameters associated to the FMS flexibility, such as:

- They should be specified and derived according to the methodology proposed for the analysis of the flexibility.

- They should incorporate structural and operational aspects of the FMS.

- They should incorporate and accumulate the human expert's knowledge.

Of the models, we can conclude that the problem of the Flexibility is analyzed starting from one of the three big current problems affecting the FMS [CALINESCU et al 2003]:

- Making Decisions Problem, analyzed by the methods based on Mathematical Programming and Fuzzy Logic.

- Structural Problem, analyzed by the method of the Disjunctive Graph and

- Behavior Problem, analyzed by the method of the Temporary Constraints Network. 


\section{References}

[1] Alfonso, M., An integration model of Temporary Constraints based on methodology CLOSURE and CSP: Application to Scheduling Problems, Doctoral Thesis presented at University of Alicante, Spain, 2001.

[2] Barber, F., "Reasoning on interval and point-based disjunctive metric constraints in temporal contexts". Journal of Artificial Intelligence Research, pp:35-86, 2000.

[3] Beck, J. and Fox M., "Constraint directed techniques for scheduling alternative activities". Journal of Artificial Intelligence, pp: 211-250, 2000.

[4] Benjaafar, S. and Ramakrishman, R., "Modeling, measurement and evaluation of sequencing flexibility in manufacturing systems", International Journal of Production Research, Vol. 34, pp:11951220, 1996

[5] Chandra, P. and Tombak, M., Models for the evaluation of routing and machine flexibility, Technical Report of Decision Craft Analytics, www.decisioncraft.com, 1993.

[6] Deshmukh, A., Talavage, J. and Barash, M., "Complexity in Manufacturing Systems", IIE Transaction on Manufacturing Systems, Vol 30, pp: 645 - 655, 2002.

[7] Fortemps, P., "Introducing Flexibility in Scheduling: The Preference Approach", Advances in Scheduling and Sequencing under Fuzziness, Ed. Springer Verlag, pp:61-79, 2000.

[8] Gupta, A., "Approach to Characterize Manufacturing Flexibility", Second World Conference on Production \& Operations Management Society, Cancun - México, pp:100-120, 2004.

[9] Calinescu, A., Sivadasan, S., Schirn, J. and Huaccho, L., Complexity in Manufacturing: An Information Theoretic Approach, Technical Report of Manufacturing System Research Group, Department of Engineering Science, University of Oxford, England, 2003.

[10] Pelaez, J. and Ruiz, J., Measuring Operational Flexibility, Technical Report, University of Murcia, Faculty of Economy and Enterprise, Spain, 2004.

[11] Sethi, A. and Sethi, S., "Flexibility in Manufacturing: A survey", International Journal of Operations and Production Management, pp:35-45, 1992.

[12] Tsourveloudis, N. and Phillis, Y., "Fuzzy Assessment of Machine Flexibility", IEEE Transaction on Engineering Management, Vol. 45, pp:78-87, 1998.

Héctor Kaschel C. and Luis Manuel Sánchez y Bernal Depto. de Ingeniería Eléctrica and Depto. de Matemática y Ciencia de la Computación Universidad de Santiago de Chile

Avda. Ecuador $\sharp 3519$ Estación Central. Santiago - Chile E-mail: hkaschel@lauca.usach.cl o msanchez@lauca.usach.cl 